\title{
DERRIDA ET LE TEMPS HUSSERLIEN
}

\author{
Daniel Giovannangeli \\ Unité de recherche «Phénoménologies» \\ Université de Liège
}

On peut considérer l'exposé que je vais faire comme un commentaire du chapitre V de La Voix et le phénomène (1967). Jacques Derrida y insère une lecture des Leçons sur le temps de Husserl (1905) dans le cours de l'examen qu'il effectue de la première des Recherches logiques. Cette lecture opère - mais Derrida déroge-t-il jamais à cette démarche que je retrouverai plusieurs fois? - selon un double mouvement. D'une part, elle rapporte Husserl à ce que Derrida désigne, ou plutôt à ce qu'il a provisoirement désigné avant de le nuancer, comme la métaphysique de la présence. Plus spécifiquement, elle marque l'appartenance de la phénoménologie husserlienne à la métaphysique de la présence sous sa forme moderne: la présence à soi de la conscience de soi. D'autre part, elle libère ce qui se dérobe chez Husserl à cette métaphysique de la présence.

Auparavant, en 1962, dans son Introduction à L'Origine de la géométrie de Husserl, Derrida avait exhibé la profonde solidarité, au sein de la temporalité phénoménologique originaire, entre l'Absolu phénoménologique du Présent vivant et l'Idée au sens kantien qui l'unifie: "[..] Le Présent Vivant de la conscience ne se retient lui-même comme Absolu primordial que dans une protention indéfinie, anịmée et unifiée par l'Idée (au sens kantien) du flux total du vécu ». ${ }^{1}$ Le Présent Vivant, risque Derrida, est une Maintenance, la Main-

\footnotetext{
1 J. Derrida, Introduction à L'Origine de la géométrie, Paris: PUF, 1962, pp. 148-149. Je dois renoncer ici à esquisser l'analyse que demanderait le texte de 1931, dont Jean-François Lavigne a donné la traduction, dans lequel Husserl comprend la question du Présent Vivant comme celle de «la possibilité transcendantale de l'expérience du même comme le même» (E. Husserl, «Le monde du présent vivant et la constitution du monde ambiant extérieur à
} 
tenance dans la rétention d'un Maintenant tendu vers un Maintenant prochain. Prenant à la lettre l'expression Présent Vivant, soulignant que l'unité ouverte à l'avenir de la conscience temporelle «s'annonce dans le Présent» et constitue pour la conscience «une possibilité vécue», Derrida conclut qu'à l'opposé, «la mort ne serait pas comprise comme sens, mais comme fait extrinsèque au mouvement de la temporalisation $"{ }^{2}$ Creusant et compliquant cette veine, La Voix et le phénomène met en relation stricte l'idéalité, le présent vivant et la vie transcendantale: «La phénoménologie, métaphysique de la présence dans la forme de l'idéalité, est aussi une philosophie de la vie». ${ }^{3}$.Je commencerai par prélever dans cette perspective quelques passages du livre.

Dans l'Introduction de La Voix et le phénomène, Derrida voit dans l'idéalité le thème central de la pensée de Husserl. Plus précisément, il y dévoile la prémisse phénoménologique - à savoir «la présence d'un objet quelconque à la conscience dans l'évidence claire d'une intuition remplie ou de la présence à soi dans la conscience, "conscience" ne voulant rien dire d'autre que la possibilité de la présence à soi du présent dans le présent vivant ». ${ }^{4}$ À son autre bout, le livre s'achèvera par un diagnostic: 1 'impossibilité du principe des principes husserlien: «Et contrairement à ce que la phénoménologie - qui est toujours phénoménologie de la perception - a tenté de nous faire croire, contrairement à ce que notre désir ne peut pas ne pas être tenté de croire, la chose même se dérobe toujours $1 .^{5}$ Prise au sérieux, cette conclusion est lourde de conséquences pour la phénoménologie husserlienne, puisqu'elle touche à sa racine. On peut ajouter aujourd'hui qu'elle distingue carrément la position derridienne d'avec celle de Heidegger, dont Dominique Pradelle a montré qu'il «opère une radicalisation du principe intuitionniste $»{ }^{6} \mathrm{Il}$ est frappant que La Voix et le phénomène - dont le sous-titre est «Introduction au problème du signe dans la phénoménologie de Husserl» - transpose au signe ce que l'Introduction à L'Origine de la géométrie considérait comme la détermination extrinsèque de la mort par rapport à la temporalité vécue. Au chapitre IV, «Le vouloir-dire et la représentation », Derrida, dans un passage qu'il faudrait citer plus longuement et plus complètement encore que je ne le fais, ramène le principe des

la chair», dans La Terre ne se meut pas, tr. fr. D. Franck, D. Pradelle et J.-F. Lavigne, Paris: Minuit, 1989, p. 80).

2 J. Derrida, ibid, p.150.

3 J. Derrida, La Voix et le phénomène, $2^{\mathrm{e}}$ éd. corrigée, Paris: PUF, coll. "Quadrige», 1998, $1^{\text {ère }}$ éd. 1967, p. 9.

4 J. Derrida, ibid., p. 8.

5 Ibid., p. 117.

6 D. Pradelle, L'Archéologie du monde. Constitution de l'espace, idéalisme et intuitionnisme chez Husserl, Dordrecht: Kluwer, 2000, p. 203. 
principes au présent vivant avant de lui objecter la médiation structurelle du signe, elle-même audacieusement rapportée à la mort: «Que signifie en effet le "principe des principes" de la phénoménologie? Que signifie la valeur de présence originaire à l'intuition comme source de sens et d'évidence, comme a priori des a priori? Elle signifie d'abord la certitude, elle-même idéale et absolue, que la forme universelle de toute expérience (Erlebnis) et donc de toute vie, a toujours été et sera toujours le présent. Il n'y a et il n'y aura jamais que du présent. L'être est présence ou modification de présence. [...] Penser 'la présence comme forme universelle de la vie transcendantale, c'est m'ouvrir au savoir qu'en mon absence, au-delà de mon existence empirique, avant ma naissance et après ma mort, le présent est. [...] C'est donc le rapport à ma mort (à ma disparition en général) qui se cache dans cette détermination de l'être comme présence, idéalité, possibilité absolue de répétition. La possibilité du signe est ce rapport à la mort. La détermination et l'effacement du signe dans la métaphysique est la dissimulation de ce rapport à la mort qui produisait pourtant la signification $\rangle^{7}$

La version huśserlienne de la métaphysique de la présence n'en est qu'une parmi d'autres. La modernité accomplit en effet le privilège de la présence pleine, inentamée, sous la modalité de la présence à soi. Le chapitre VI («La voix qui garde la présence») la resserre autour du concept d'autoaffection. Ce concept, Derrida l'importe explicitement du livre de Heidegger sur Kant et le problème de la métaphysique, pour désigner le mouvement de la temporalisation que décrivent les Leçons de Husserl sur le temps: "Le processus par lequel le maintenant vivant, se produisant par génération spontanée, doit, pour être un maintenant, se retenir dans un autre maintenant, s'affecter lui-même, sans recours empirique, d'une nouvelle actualité originaire dans laquelle il deviendra non-maintenant comme maintenant passé, etc., un tel processuis est bien une auto-affection pure dans laquelle le même n'est le même qu'en s'affectant de l'autre, en devenant l'autre du même ". $^{8}$ Comme on le pressent là, Derrida s'emploie alors à montrer, en deux moments, comment Husserl affirme, d'une part, le privilège de l'auto-affection comme rapport de soi à soi pour, d'autre part, reconnaître la nécessaire contamination de cette pureté par l'hétéro-affection et la discontinuité. Sans plus de nuance, j'enchaine, en citant le texte derridien, ces deux moments de l'argumentation déconstructive: 1. «Cette auto-affection doit être pure puisque l'impression originaire n'y est affectée par rien d'autre que par elle-même, par la "nou-

7 J. Derrida, La Voix et le phénomène, op.cit., pp. 59-60. De là, dans un horizon heideggerien: «Je suis veut donc dire originairement je suis mortel» (p. 60).

8 J. Derrida, Ibid., pp. 94-95. 
veauté" absolue d'une autre impression originaire qui est un autre maintenant» $;{ }^{9}$ 2. «Mais cette différence pure, qui constitue la présence à soi du présent vivant, y réintroduit originairement toute l'impureté qu'on a cru pouvoir en exclure $\gg .{ }^{10}$ Ainsi procède en deux temps, stratégiquement, la déconstruction, - le terme figure déjà dans La Voix et le phénomène -, qui retrouve dans un second moment, jusqu'au cœur même de la présence à soi dans l'autoaffection, cela même, cette altérité, que la présence à soi s'efforçait d'exclure: l'espace, le dehors, le monde, etc., bref, ce que Derrida subsumait à cette époque sous le terme en quelque sorte générique de «différence».

Une précaution s'impose encore. Par méthode, je m'en tiens au cadre que je viens d'esquisser. Mais il convient que j'indique que pour Derrida, dès lors qu'il y va du temps, il y va de l'autre. L'autre et le temps se soustraient également, d'un même mouvement, au principe des principes husserliens, à la juridiction de l'intuitivité de la chose, de l'étant-présent dans sa présence vivante, c'est-à-dire à la présence à soi de la vie transcendantale. Derrida associe d'un trait la résistance que les descriptions husserliennes de la temporalité et de l'intersubjectivité dressent devant le principe des principes: «Notons seulement, pour préciser ici notre intention, que la phénoménologie nous parait tourmentée sinon contestée de l'intérieur par ses propres descriptions du mouvement de la temporalisation et de la constitution de l'intersubjectivité. Au plus profond de ce qui lie ensemble ces deux moments décisifs de la description, une non-présence irréductible se voit reconnaitre une valeur constituante, et avec elle une non-vie ou une non-présence ou une nonappartenance à soi du présent vivant, une indéracinable non-originalité». ${ }^{11}$ La notion de différance - elle aussi, déjà à l'œuvre dans La Voix et le phénomène - visera à condenser ces deux motifs que Derrida estime irréductibles à la phénoménologie en son principe des principes. S'agissant de la constitution de l'intersubjectivité, le concept d'Appräsentation thématise chez Husserl l'altération, la modification de la présence à soi. L'objectivité trouve sa condition de possibilité dans l'intersubjectivité. Si, dirais-je, l'identité d'un monde commun résulte de la différence, la phénoménologie transcendantale ne peut se prémunir de la contamination de l'auto-affection par l'hétéro-affection, elle ne peut, structurellement, faire l'économie d'un détour, d'un "passage nécessaire par l'apprésentation dans le rapport à l'alter ego, c'est-à-dire dans le rapport à ce qui rend possible aussi une objectivité idéale en général, l'intersubjectivité étant la condition de l'objectivité et celle-ci n'étant absolue que

\footnotetext{
9 J. Derrida, ibid., p. 95.

10 J. Derrida, ibid., p. 95.

11 J. Derrida, ibid., p. 5.
} 
dans le cas des objets idéaux $\$ .{ }^{12}$ D'une manière, il n'y aurait rien d'aberrant à poser que le projet de Derrida en son commencement était animé par le désir de reprendre à son compte, et à nouveaux frais, l'ambition husserlienne d'élaborer une esthétique transcendantale. Dans une communication de jeunesse, dès 1959, Derrida évoquait d'ailleurs «cette nouvelle esthétique transcendantale" qui sera sans cesse annoncée mais toujours différée, et dans laquelle les thèmes de 1'Autre et du Temps devaient laisser apparaitre leur irréductible complicité $\gg .{ }^{13} \mathrm{On}$ m'accordera qu'il n'est pas insignifiant que le même thème fasse retour bien plus tard, en 2000, dans le livre, encore peu étudié d'un point de vue phénoménologique, sur Le Toucher, où l'on peut lire: «Noụs sommes ici dans la zone de l'immense problème de l'intersubjectivité phénoménologique (de l'autre et du temps) que nous ne re-déploierons pas, une fois de plus, pour elle-même. Qu'il nous suffise de relancer ici la question: une certaine intropathie introjective, une certaine " intersubjectivité" ne doit-elle pas avoir déjà introduit l'autre et l'apprésentation analogique dans le touchant-touché pour que celui-ci donne lieu à une expérience du corps propre qui permette de dire "c'est moi", "ceci est mon corps"? ».14 De bout en bout de l'œuvre derridienne, il y va, on peut le constater, de la contamination de l'auto-affection pure par l'hétéro-affection.

\section{Husserl, Derrida, et la question du temps}

Dans ses Leçons de 1905, Husserl consacre un long développement ${ }^{15}$ à «la théorie de Brentano sur l'origine du temps». Brentano y est crédité par Husserl d'avoir cerné avec justesse le rôle que la modification temporelle joue dans la constitution de la durée vécue. Ce «noyau phénoménologique»; Husserl le conserve intact: «De la durée, de la succession, des changements apparaissent. Qu'y a-t-il dans cet apparaitre? Dans une succesșion, par exemple,

\section{J. Derrida, ibid., p. 5.}

$13 \mathrm{~J}$. Derrida, L'Écriture et la différence, Paris : Le Seuil, 1967, p. 244.

14 J. Derrida, Le Toucher: Jean-Luc Nancy, Paris: Galilée, 2000, p. 202. Sur ce livre, on lira C. Malabou, "Gré», dans Critique, 636, mai 2000, pp. 440-456, le compte-rendu d'A.Van Sevenant dans la Revue internationale de philosophie, 213, 2000, pp. 515-523, et, s'agissant plus particulièrement de la lecture que Derrida y fait de Merleau-Ponty, R. Gély, «Le pli et le toucher à distance chez Merleau-Ponty, dans Gr. Cormann, S. Laoureux et J. Piéron (éds.), Différence et identité. Les enjeux phénoménologiques du pli, Hildesheim: Olms, 2006, pp. 141-160.

$15 \S \S 3-6$. 
apparait un "maintenant" et, formant une unité avec lui, un "passé"》». Cette unité que Brentano dégage, cette unité de la conscience qui embrasse tout ensemble le présent et le passé, correspond aux yeux de Husserl à un authentique donné phénoménologique.

Le désaccord de Husserl vis-à-vis de Brentano surgit lorsqu'il s'agit d'expliquer cette unité synthétique (Husserl parlera plus tard de synthèse passive) du présent et du passé. L'origine des modifications temporelles réside, selon Brentano, dans l'imagination productrice. Les prédicats temporels modificateurs sont, pour Brentano, irréels. Ce qui revient à estimer que pour Brentano, cette mélodie que nous écoutons, nous croyons ${ }^{17}$ (wir glauben, dit Husserl) donc l'entendre comme nous croyons percevoir le tout-juste passé. Autrement dit, ce ne seraient donc là, s'inquiète Husserl, qu'apparences. Faire résider, avec Brentano, l'origine du passé vécu dans l'imagination est lourd de conséquences. Si la perception du changement et de la succession est niée, alors notre champ temporel est tout entier de l'ordre de l'imaginaire.

À l'aporie dans laquelle Brentano lui semble s'enfermer en niant la perception du changement et en rapportant la durée à l'imagination, Husserl se soustrait, au $\S 17$ des Leçons, en distinguant le souvenir primaire ou rétention, du souvenir secondaire ou reproduction. Ce dernier, le souvenir secondaire, c'est-à-dire la reproduction, ressortit bien au domaine de l'imagination. Mais, corrige Husserl, le souvenir primaire, la rétention du tout-juste passé, est, lui, de l'ordre de la perception: «[...] Si nous nommons perception l'acte en qui réside toute origine, l'acte qui constitue originairement, alors le souvenir primaire est perception. Car c'est seulement dans le souvenir primaire que nous voyons le passé, c'est seulement en lui que se constitue le passé, et ce non pas de façon représentative, mais au contraire présentative $\gg{ }^{18}$ Bref, la rétention est perception; elle est la perception, présente par définition, du toutjuste passé. La ligne de démarcation passe ainsi entre le souvenir primaire, qui est de l'ordre de la perception, et le souvenir secondaire, qui est, lui, de l'ordre de l'imagination. La solution que Husserl apporte à la difficulté où Brentano lui paraissait s'enfermer consiste à étendre la perception à un perçu qui n'est pas présent, qui n'est plus ou n'est pas encore présent.

Jacques Derrida a reconnu la dette qu'il a contractée vis-à-vis de cette conception phénoménologique du temps. L'avant-propos qu'il a donné à l'édition, en 1990, de son mémoire de fin d'études de 1953-1954, Le Problème

${ }^{16}$ E. Husserl, Leçons pour une phénoménologie de la conscience intime du temps, tr. fr. H. Dussort, Paris: PUF, 1964, p. 25.

17 E. Husserl, ibid., p. 22.

18 E. Husserl, ibid., p. 58. 
de la genèse dans la philosophie de Husserl, signale le rôle important qu'y joue l'interprétation du temps husserlien dans une perspective dialectique. Le recours à la dialectique visait à l'époque à surmonter l'obstacle que l'irréductibilité du temps constitué oppose à la recherche d'une origine constituante du temps. Derrida soulève la difficulté qu'entrainent les modifications rétentionnelle et protentionnelle pour la conception husserlienne de l'impression originaire. En dépit de l'intention expresse de Husserl, ne conduisent-elles pas à renoncer au principe d'une impression originaire? À reconnaitre, du moins, que le présent originaire est lui-même déjà constitué? ${ }^{19}$

$\mathrm{Au} \S 3$ des Leçons de 1905, Husserl tirait de la description de la mélodie des enseignements pour une phénoménologie du temps. Il remarquait que, pas plus qu'elle n'admettrait sans se détruire que l'apparition d'un son entraine la disparition complète du son précédent, la mélodie ne pourrait supporter, sauf à se confondre avec une véritable cacophonie, que chacun des sons passés se conserve sans modification dans la conscience actuelle. L'unité de la mélodie éclaire l'unité synthétique de la temporalité. Si chaque son s'effaçait brutalement devant le son qui lui succède, on n'aurait pas de mélodie: tout au plus une succession de sons isolés et d'intervalles de silence. $\mathrm{Si}$, au contraire, les sons passés se conservaient intégralement, ils se superposeraient les uns aux autres dans la simultanéité. Nous n'aurions pas davantage de mélodie: tout au plus, une cacophonie. La solution réside dans la modification: les sons passés se conservent dans le présent, mais en se modifiant: «Lorsqu'un son nouveau retentit, le précédent n'a pas disparu sans laisser de trace, sinon nous serions bien incapables de discerner les relations entre sons qui se suivent l'un l'autre: nous n'aurions à chaque instant qu'un seul son, éventuellement dans l'intervalle de temps entre le tintement de deux sons une phase vide, mais jamais la représentation d'une mélodie. D'un autre côté, il ne peut s'agir de faire demeurer les représentations des sons dans la conscience. Si elles y demeuraient en effet sans modification, nous aurions alors, au lieu d'une mélodie, un accord fait de sons simultanés, ou plutôt une cacophonie comme nous pourrions en obtenir une si tous les sons qui ont déjà résonné, du plus loin que ce soit, retentissaient tous à la fois. C'est seulement parce qu'intervient cette modification spécifique, qui veut que chaque sensation de son, après la disparition de l'excitation qui l'a engendrée, éveille d'elle-même une représentation semblable et munie d'une détermination temporelle, et parce que cette modification temporelle se transforme continument, que peut avoir lieu

19 J. Derrida, Le Problème de la gènèse dans la philosophie de Husserl, Paris: PUF, 1990, p. 128. 
la représentation d'une mélodie, en qui les sons individuels ont chacun leur place déterminée et leur mesure temporelle déterminée».20

Au jeune Derrida lecteur des Leçons husserliennes sur le temps, la question se pose d'emblée de savoir si la nécessité de la modification rétentionnelle (et protentionnelle) reste conciliable avec l'originalité de l'impression. $\mathrm{Au} \S 31$, Husserl s'efforçait de conserver l'impression originaire pure de toute modification en lui conférant le statut du «non-modifié absolu». Cependant, il repoussait presque aussitôt, au $\S 32$, l'éventualité d'un «maintenant que rien n'aurait précédé». N'était-ce pas, en préservant la nécessité de la rétention, contrarier la valeur d'originalité de l'impression présente? S'il n'est de présent que constitué, si chaque présent reste inséparable du présent passé qu'il retient, l'idée ne s'imposerait-elle pas, intuitivement paradoxale, d'un retard originaire de la conscience? Cette idée paradoxale, Husserl s'y refusait explicitement au $\S 13$, et il jugeait phénoménologiquement inconcevable «une conscience initiale, débutant par un souvenir récent, sans avoir eu de perception préalable».

Au contraire, le mémoire de Derrida sur Le Problème de la genèse assume cette possibilité, écartée par Husserl, d'un retard originaire de la conscience. La solution que Derrida apporte à cette aporie dans son premier ouvrage est en quelque sorte du ressort d'une ontologie phénoménologique. Admettre, avec Husserl, que l'instant de l'impression originaire suppose d'ores et déjà la rétention, reviendrait à introduire au cœur de la conscience constituante, avec l'antériorité, la transcendance de la chose constituée.: «[...] Quelle différence essentielle y a-t-il entre la transcendance des moments constitués à l'intérieur du flux pur du vécu par rapport à un " maintenant" originaire et la transcendance des objectivités "réelles" du temps?»."1

Husserl remarque d'ailleurs lui-même que la réflexion introduit un dédoublement qui interdit la coïncidence du constituant et du constitué: "Ce qui, dans l'actualité instantanée du flux de conscience, est amené à apparaitre, c'est une phase passée de ce même flux dans la suite de ses moments rétentionnels $).{ }^{22}$

Dans cet entremêlement du constituant et du constitué, Derrida décelait à cette époque comme la force d'une nécessité interne qui poussait la phénoménologie à s'ouvrir à l'ontologie qu'elle entendait pourtant suspendre. Husserl aurait beau prétendre s'en tenir à la pureté de l'origine constituante du temps; si, dans le fait, il ne pouvait empêcher le retour du constitué dans le constituant,

20 E. Husserl, Leçons..., op. cit., pp. 19-20.

21 J. Derrida, Le Problème de la genèse dans la philosophie de Husserl, op.cit, p. 111.

22 . E. Husserl, Leçons..., op. cit., p. 109. 
«la phénoménologie ne serait plus tout à fait maitresse chez elle. L'ontologie serait déjà dans la place». La rétention du passé renverrait ainsi à la facticité du monde. Une commune antériorité imposerait à la conscience et le monde et le passé qu'elle retient. En d'autres termes, la constitution dépendrait du donné qu'elle conserve en le dépassant en quelque sorte dialectiquement: "Quand Husserl reconnait une "nécesșité a priori de l'antécédence d'une impression à toute rétention" et que, d'autre part; il affirme que la rétention présente originairement un caractère d'évidence intentionnelle, ne réintroduit-il pas, sous la forme du "datum hylétique" passivement reçu, l'objet transcendant qu'il prétendait exclure de ses analyses ? $\gg .^{23}$ Pour tenter de resserrer lapidairement cette conclusion que le jeune Derrida arrache à Husserl à son corps défendant, je dirais que le retard dans la conscience est inséparable du retard de la conscience sur l'être.

\section{Une dialectique ontologique du temps}

Cette interprétation par le tout premier Derrida du retard en termes d'ontologie phénoménologique, comment ne pas remarquer qu'elle parait épouser, et même fonder sur nouveaux frais, le mouvement de la pensée sartrienne? Sartre, à bien y regarder, renvoie l'être à la facticité et la facticité à la temporalité dans sa dimension passée. C'est à l'être en soi que $L$ 'Être et le néant identifie la facticité, pour conclure que «facticité et passé sont deux mots pour désigner une seule et même chose». ${ }^{24}$ Pour dire sans nuance ce que j'ai essayé de nuancer ailleurs, ${ }^{25} 1$ 'ontologie phénoménologique sartrienne prend acte du retard de la conscience sur l'être. L'antériorité de l'être en soi sur le pour-soi (et qui amène Sartre à parler, presque indifféremment, non seulement de l'être transphénoménal du phénomène mais aussi de l'être transphénoménal de la conscience) est la solution que cette ontologie phénoménologique, décidée à refuser toute légitimité à l'altérité de l'inconscient, apporte à l'épreuve que la conscience fait de son retard originaire. Plus exactement, l'être non conscient de l'en-soi est ce qui se substitue et tout à la fois se préserve de l'inconscient: «[... La conscience implique dans son être un être non conscient et transphénoménal $[. .$.$] Dire que la conscience est conscience de quelque chose, c'est$

23 J. Derrida, Le Problème de la genèse dans la philosophie de Husserl, op. cit., p. 121.

24 J.-P. Sartre, L'Être et le néant, Paris: Gallimard, 1943, p.162.

${ }^{25}$ Cf. D. Giovannangeli, Le Retard de la conscience. Husserl, Sartre, Derrida, Bruxelles: Ousia, 2001. 
dire qu'elle doit se produire comme révélation-révélée d'un être qui n'est pas elle et qui se donne comme existant déjà lorsqu'elle le révèle ${ }^{26}$

Il ne s'agit pas pour autant de simplifier le rapport que le premier Derrida établit entre phénoménologie et ontologie en s'autorisant de cette proximité à l'ontologie sartrienne. Il est certain que la brèche opérée par La Transcendance de l'ego n'a pas échappé à Jacques Derrida. L'Introduction à L'Origine de la géométrie définit l'écriture comme un champ transcendantal sans sujet: en tant qu'elle virtualise le dialogue, l'écriture forge une sorte de champ transcendantal dont le sujet peut s'absenter (c'est l'idée, sur laquelle MerleauPonty est souvent revenu, suivant laquelle le sujet transcendantal est intersubjectivité). Il n'est probablement pas insignifiant que le premier nom que le mémoire de fin d'études évoquait soit le nom de Sartre. Il reste qu'en dépit de cette proximité à Sartre, au mouvement qui préside chez celui-ci à l'approfondissement ontologique de la phénoménologie, à l'identification qui s'y accomplit de la facticité de l'en soi et du passé - en somme, de l'être passé - c'est, plutôt qu'en termes sartriens, en termes de dialectique qu'une ontologie est cernée par Derrida. Plus précisément, Le Problème de la genèse dans la philosophie de Husserl fait droit à une «dialectique ontologique du temps». En radicalisant les interprétations dialectiques de Cavaillès, d'Yvonne Picard et de Tran-Duc-Thao, Derrida fait l'hypothèse qu'il est «possible de fonder dans sa possibilité ontologique et dans son sens (à la fois) une dialectique absolue de la dialectique et de la non-dialectique». Sans rupture apparente sur ce point, en 1962, l'Introduction à L'Origine de la géométrie continuera à invoquer une dialectique de la rétention et de la protention qu'elle désignera à son tour comme la dialectique de la dialectique avec la non-dialectique, et qu'elle explicitera comme «la dialectique de la dialectique - l'implication indéfinie, mutuelle et irréductible des protentions et des rétentions - avec la non-dialectique - l'identité absolue et concrète du Présent Vivant, forme universelle de toute conscience $»{ }^{27}$

\section{De la dialectique à la «différance». Passage vers Freud}

La Voix et le phénomène, accomplit, en 1967, le retrait explicite de la dialectique au profit de la différance. Derrida y envisage la temporalité husserlienne, dialectiquement en somme, en parlant de «l'identité de l'identité et de la non-identité», pour se risquer à parler aussi, concurremment, de «la diffé-

26 J.-P. Sartre, L'Être et le néant, op. cit., p. 29.

27 J. Derrida, Introduction à L'Origine de la géométrie, Paris, op. cit., pp. 157-158. 
rance dans l'auto-affection". Il se demande si «cette "dialectique" - à tous les sens de ce mot et avant toute reprise spéculative de ce concept-n'ouvre pas le vivre à la différance $\gg .^{28}$

C'est dans cette perspective que, consommant la rupture avec la dialectique, la conférence de janvier 1968 sur «la différance» s'autorisera désormais de la Nachträglichkeit freudienne: «La structure du retardement (Nachträglichkeit) interdit en effet qu'on fasse de la temporalisation (temporisation) une simple complication dialectique du présent vivant comme synthèse originaire et incessante, constamment reconduite à soi, sur soi rassemblée, rassemblante, de traces rétentionnelles et d'ouvertures protentionnelles $\gg .{ }^{29}$ Il est vrai que, déjà, l'année précédente, La Voix et le phénomène mesurait à l'aune de l'inconscient freudien l'obstacle que dresse devant le phénoménologue la possibilité d'un retard originaire. Derrida met en évidence dans La Voix et le phénomène la façon dont Husserl, dans le Supplément IX des Leçons, s'interdit de lier la rétention à l'inconscient: «C'est, déclarait Husserl, une véritable absurdité que de parler d'un contenu "inconscient" qui ne deviendrait conscient qu'après coup (nachträglich)». La lecture derridienne met au contraire en lumière la rigoureuse nécessité qui entraîne Husserl, malgré lui, jusqu'à la Nachträglichkeit: «Ce n'est pas un hasard si les Leçons sur la conscience intime du temps confirment la dominance du présent et rejettent à la fois l'"après-coup"du devenir-conscient d'un "contenu inconscient", c'està-dire la structure de la temporalité impliquée par tous les textes de Freud». ${ }^{30}$ Pour le dire massivement, Derrida s'incline devant cette nécessité que libère sa lecture de Husserl, et il accomplit, en stricte conséquence, le pas que Husserl s'interdit et suspend. Ce faisant, en excédant les limites de la phénoménologie en son principe, il se porte, poussé par Husserl plus loin que Husserl, à la rencontre de Freud.

Dans De la Grammatologie, en 1967, Jacques Derrida manifeste franchement son éloignement par rapport à ce qu'il y désigne comme la «dialectique » de la rétention et de la protention. C'est, souligne-t-il, que la conception husserlienne de la temporalité reste fermée à la possibilité d'un présent que ne supporterait pas un présent le précédant immédiatement: «C'est le problème de l'effet à retardement (nachträglich) dont parle Freud. La temporalité à laquelle il se réfère ne peut être celle qui se prête à une phénoménologie de la conscience ou de la présence $» .{ }^{31}$

28 J. Derrida, La Voix et le phénomène, op. cit., p. 77.

29 J. Derrida, Marges de la philosophie, Paris : Minuit, 1972, pp. 21-22.

30 J. Derrida, La Voix et le phénomène, op. cit., pp. 70-71.

31 J. Derrida, De la Grammatologie, Paris : Minuit, 1967, p. 98. 
Freud rencontre, dans $A u$-delà du principe de plaisir, un problème qui, suggère Derrida, devrait être creusé. Il conviendrait de confronter l'intemporalité des processus psychiques avec la thèse kantienne - laquelle, prévient Freud, s'en trouverait fortement ébranlée - qui fait du temps l'une des formes nécessaires de notre subjectivité. Or, si la question est esquissée par Freud, Derrida l'affronte carrément. Lorsqu'il la rappelle peut-être le plus fortement, dans «Freud et la scène de l'écriture», en 1966, c'est pour la déplacer, en en reconsidérant la portée philosophique, d'un double geste.

1. D'abord (c'est son premier geste), il détache la conception freudienne d'un «retardement supplémentaire» du cadre traditionnel de la réminiscence, tant philosophique (de Platon à Hegel) que littéraire (Proust). De Freud, Derrida évoque l'Homme aux loups; il cite le passage de l'Esquisse d'une psychologie scientifique (1895) relatif au refoulement dans l'hystérie. Après avoir signalé que nachträglich signifie tout à la fois après coup et supplémentaire, Derrida écrit: «La métaphore du chemin frayé, si fréquente dans les descriptions de Freud, communique toujours avec le thème du retardement supplémentaire et de la reconstitution du sens après-coup, après un cheminement de taupe, après le labeur souterrain d'une impression. Celle-ci a laissé une trace travailleuse qui n'a jamais été perçue, vécue dans son sens au présent, c'est-à-dire en conscience. Le postscriptum qui constitue le présent passé comme tel ne se contente pas, comme l'ont peut-être pensé Platon, Hegel et Proust, de le réveiller ou de le révéler dans sa vérité. Il le produit. [...] Voyez l'homme aux loups. C'est à retardement que la perception de la scène primitive - réalité ou fantasme, peu importe - est vécue dans sa signification et la maturation sexuelle n'est pas la forme accidentelle de ce retard. "À un an et demi, il recueillit des impressions dont la compréhension différée lui fut possible à l'époque du rêve de par son développement, son exaltation et son investigation sexuelle". Déjà dans l'Esquisse, à propos du refoulement dans l'hystérie: "On découvre dans tous les cas qu'un souvenir est refoulé, qui ne se transforme en trauma qu'à retardement (nur nachträglich). La cause en est le retardement (Verspätung) de la puberté par rapport à l'ensemble du développement de l'individu"». ${ }^{32}$

2. Du rappel des textes freudiens qui font droit à la Nachträglichkeit, Derrida retient l'idée que le présent passé y est, non pas réveillé, mais en quelque sorte produit dans sa vérité. C'est dire, en d'autres termes, que les processus inconscients demeurent incommensurables avec la réminiscence philosophique. Cependant, si les processus inconscients demeurent incommensurables avec la réminiscence, ensuite ( c'est son second geste), Derrida se refuse

32 J. Derrida, L'Ecriture et la différence, op. cit., pp. 317-318. 
toutefois à précipiter la conclusion suivant laquelle les processus inconscients se déroberaient définitivement'à toute détermination philosophique: «L'intemporalité de l'inconscient n'est sans doute déterminée que par opposition à un concept courant du temps, concept traditionnel, concept de la métaphysique, temps de la mécanique ou temps de la conscience. Il faudrait peut-être lire Freud comme Heidegger a lu Kant: comme le je pense, l'inconscient n'est sans doute intemporel qu'au regard d'un certain concept vulgaire du temps . $^{33}$ Davantage, dans le commentaire derridien, publié en 1980, d'Au-delà du principe de plaisir, la référence à la temporalité phénoménologique, inquiétée, presque. congédiée, par La Voix et le phénomène, se trouve rétablie dans son originalité philosophique et sollicitée pour nuancer l'intemporalité que Freud attache à l'inconscient. Derrida y propose en effet de rapporter la temporalité freudienne à «la structure auto-affective du temps (ce qui s'y donne à recevoir n'est aucun étant-présent) telle qu'elle est décrite dans les Leçons sur la conscience interne du temps de Husserl ou le Kantbuch de Heidegger $\gg .{ }^{34}$ Derrida, s'il continue de voir dans la lecture heideggerienne de Kant une rupture par rapport à la conception vulgaire, c'est-à-dire à la conception métaphysique du temps, lui associe cette fois sans rechigner la phénoménologie husserlienne. Son appréciation des Leçons accentue l'une ou l'autre direction $\mathrm{du}$ double mouvement que $\mathrm{j}$ 'avais indiqué en commençant, du balancement qu'il repère chez Husserl, entre une prévalence de l'identité et une reconnaissance de la différence.

\section{Conclusion}

À suivre Derrida, l'écart institué par Freud à l'égard de la philosophie kantienne demanderait à être lui-même reconsidéré philosophiquement, en tout cas à partir des déterminations phénoménologiques, philosophiquement originales, de la temporalité. En somme, Freud permettrait, en dépit des déclarations expresses de Husserl, d'accomplir Husserl au-delà des limites que celui-ci se traçait par principe. Dans la mesure où elle se libère de la primauté de l'étant-présent, la temporalité phénoménologique ne fait-elle pas place nette pour une autre figure du temps, dont la spécificité légitimerait, en dépit de Freud, l'idée d'une temporalité de l'inconscient? Le rejet, déclaré par Freud, d'une temporalité de l'inconscient ne vaudrait alors que contre la conception vulgaire du temps dont Husserl et Heidegger auraient précisément

33 J. Derrida, ibid., p. 318. 34 J. Derrida, La Carte postale. De Socrate à Freud et au-delà, Paris: Flammarion, 1980, p.
382 . 
délivré la philosophie. Il faudrait mesurer la portée philosophique de ce déplacement. Quand, dans son essai de 1965 sur Freud, De l'Interprétation, Paul Ricœur rappelait que l'intemporalité caractérise l'inconscient, dès avant la Métapsychologie, ${ }^{35}$ il notait que son intemporalité soustrayait dẹ la sorte l'inconscient à l'emprise d'une esthétique transcendantale: «Si l'inconscient est intemporel (zeitlos), c'est qu'il n'a pas proprement rapport au temps: nous sommes en deçà d'une Esthétique transcendantale; "le rapport temporel, nous dit Freud, est lié au travail du système conscient"». ${ }^{36}$ Reprise sous cet angle, la question serait de déterminer dans quelle mesure le geste derridien, en dépit de sa critique du présent vivant, restaurerait peu ou prou, non sans s'y refuser expressément, la légitimité de l'esthétique transcendantale.

\section{ABSTRACT}

The author examines the several phases of Derrida's interpretation of Husserl phenomenology of time consciousness. The main issue is a -non-husserlian-thesis about the deferred action of primordial impression, always intermingled with retention, allowing a critique of Husserl's metaphysics of presence, and an approximation with Freud's views about the Nachträglichkeit structure of psychic life. Consequently, the author stresses that Freud will allow, despite Husserl's explicit assertions, a completion of phenomenology beyond the limits Husserl imposes on it (and on himself), as he remains dominated by the assumption of the absolute priority of primordial impression over retention.

${ }^{35}$ Cf. P. Ricœur, De l'Interprétation. Essai sur Freud, Paris : Le Seuil, coll. «Points-Essais», 1995, p. 116: «Ainsi la première fonction de la topique est de répartir de façon figurée les degrés de profondeur du désir, jusqu'à l'indestructible. Nous pouyons peut-être dire déjà que la topique est la figure métaphorique de l'indestructible en tant que tel: "Dans l'inconscient, rien ne finit, rien ne passe, rien n'est oublié". On songe déjà aux formules de la Métapsychologie: l'inconscient est hors le temps. La topique est le lieu qui figure le "hors le temps"»".

36 P. Ricœur, ibid., p. 159. 